\title{
Correlation between changes in intraocular pressure and refractive error indices post Cataract Surgery
}

\author{
Muhammad Afzal Bodla', Ali Afzal Bodla², \\ Ayema Moazzam ${ }^{3}$, Noor Tariq ${ }^{4}$
}

\begin{abstract}
Objective: To evaluate the correlation between refractive errors and change in intraocular pressure in patients undergoing cataract surgery.

Methods: This interventional retrospective case study was carried out from September 2018 to April 2019 at Bodla Eye Care and Multan Medical and Dental College, Multan. A total of 127 eyes were recruited in the study among which six were excluded. Out of remaining 121, 53 eyes were emmetropes, 41 were mild myopes and 27 were high myopes. Single surgeon performed the procedure. Pre-operative investigations of IOP and refractive error were done by goldmann tonometry and auto refractometry. IOP was reviewed at day $1,7,14$ and 28 post cataract surgeries.

Results: Out of 121 eyes, 53 eyes were emmetropes, 41 were mild myopes and 27 were high myopes, who underwent phacoemulsification. There was an elevation of $2-3 \mathrm{~mm} \mathrm{Hg}$ at Day-1, in emmetropes and mild myopes, and further on, a constant drop was noticed on follow ups. In high myopes a significant fluctuation of IOP was noted in first fourteen days followed by an unremarkable gradual decline afterwards.

Conclusion: Cataract surgery helps lowering the IOP in patients with refractive errors. Mild myopic and emmetropic patients showed a linear swift pattern while high myopes presented instable and gradual reduction in IOP. A total decrease of $1-2 \mathrm{~mm} \mathrm{Hg}$ was seen at the end of the study depicting that relation between IOP and cataract surgery is insignificant.
\end{abstract}

KEYWORDS: Cataract surgery, Intraocular pressure, Phacoemulsification, Refractive errors.

How to cite this:

doi: https://doi.org/10.12669/pjms.36.3.1597

Bodla MA, Bodla AA, Moazzam A, Tariq N. Correlation between changes in intraocular pressure and refractive error indices post Cataract Surgery. Pak J Med Sci. 2020;36(3):574-577. doi: https://doi.org/10.12669/pjms.36.3.1597

This is an Open Access article distributed under the terms of the Creative Commons Attribution License (http://creativecommons.org/licenses/by/3.0), which permits unrestricted use, distribution, and reproduction in any medium, provided the original work is properly cited.

1. Muhammad Afzal Bodla, DORCS.

2. Ali Afzal Bodla, FRCS.

3. Ayema Moazzam,

4. Noor Tariq,

1-4: Department of Ophthalmology,

Multan Medical and Dental College

Multan, Pakistan.

Correspondence:

Dr. Ali Afzal Bodla, MD, MRCOpth, MRCSEd, FRCS Associate Professor of Ophthalmology,

Multan Medical and Dental College and Bodla Eye Care, Multan, Pakistan.

Email: aliafzal111@gmail.com

* Received for Publication:

* $1^{\text {st }}$ Revision Received:

* $2^{\text {nd }}$ Revision Received:

* Final Revision Accepted:
July 31, 2019

September 3, 2019

December 30, 2019

January 17, 2020

\section{INTRODUCTION}

Cataract is an opacification in the lens of the eye interfering with visual functions, ${ }^{1,2}$ with it being the cause of $39 \%$ of blindness and $33 \%$ of severe visual impairments across the world whereby the pervasiveness in age-related cataract cases seems to be increasing. For years the cataract surgery has been performed and is still manifested beneficial in visual improvement. ${ }^{3}$ Cataract surgery was suggested to decrease intraocular pressure (IOP) in the eyes with or without glaucoma, in addition to removing the opacified lens., ${ }^{4,5}$ and after the success of cataract surgery it has therefore been preferred for glaucoma patients. ${ }^{6}$ 
Various elements that affect the intraocular pressure in patients after a cataract surgery have been studied. ${ }^{7}$ Sufficient information indicates that cataract surgery has a long-term reduction impact on IOP. This effect appears to be proportional to preoperative IOP. Eyes with greater preoperative IOP have the largest average decrease, whereas eyes with IOP in the reduced range of statistically normal range tend to have an IOP that stays unchanged from baseline or maybe higher after cataract surgery.

In patients with narrow angles, the IOPlowering effect appears to be proportional to the degree of anterior chamber deepening induced by cataract surgery. ${ }^{8}$ Various refractive errors have subsequent effects on Intraocular Pressure, post cataract; myopia for instance, being the most common cause.

However, Myopia, the most common type of refractive error, is a complex trait including both genetic and environmental factors ${ }^{9}$ Progressive myopia can lead from the sclera's hereditary biomechanical weakness that enables the sclera to stretch in reaction to stress. The objective of this study was to determine whether there is any significant lowering of intraocular pressure post cataract surgery in normotensive patients. Moreover, authors are inclined to determine whether a specific refractive error contributes more to this phenomenon.

\section{METHODS}

This was an interventional retrospective case analysis of 127 eyes that went through cataract surgery in Bodla Eye Care and Multan Medical and Dental College carried out from September 2018 to April 2019 (Ref. MMDC-, dated September 4 , 2018). Six of them being excluded because of set inclusion criteria were patients of age 20 years \& older, willing and able to understand and sign consent form and attend post-operative follow-ups as per schedule. These patients should have been free of any other ophthalmic disease, e.g. glaucoma, ARMD etc. Candidates with hypermetropia, ocular hypertension, systemic diseases, eye diseases other than cataract such as glaucoma, conditions affecting IOP and those who underwent extracapsular cataract extraction (ECCE) were excluded. Informed written consent was obtained from all subjects who participated in this study. The confidentiality of the participants was maintained for the study. The data was collected by four follow-up sessions including preoperative visit to the surgeon. Sample included patients who were myopic, were diagnosed of cataract and received phacoemulsification. Surgeries were performed by same surgeon (Bodla, AA) and all the materials were kept same to avoid disparity. Patient's bio-data (age, sex and general physical examination), preoperative examination (refractive error via Auto Refraction and Intraocular Pressure via goldmann tonometry) and postoperative examinations (refractive conditions and intraocular pressure on day one, seven, 14 and 28 were noted. Refractive error were classified 0 to $-0.25 \mathrm{D}$ as emmetropia, $-0.5 \mathrm{D}$ to $-5.75 \mathrm{D}$ as mild to moderate myopia and -6 \& greater as high myopia. Analysis was done using SPSS software version 20.0. Data was evaluated using descriptive statistics and relation with variables was calculated via Chi-square test. A value $\mathrm{P}<0.2$ in the analysis was considered as a variable selection criterion.

\section{RESULTS}

One hundred twenty-one eyes underwent phacoemulsification at Bodla Eye Care \& MMDC in period of September, 2018 - April, 2019, out of which '53' were emmetropes, ' 41 ' were mildmoderate myopes \& ' 27 ' were high myopes. They all embraced the criteria that were set. Surgeon performing surgery (Bodla, AA) \& intraocular lens implanted were kept same. IOP was statistically insignificant in relation to the refractive errors. $(\mathrm{P}=0.011)$.

IOP at Day 1 in patients with emmetropia and mild myopia, after surgery was slightly elevated but $(\mathrm{P}=0.105)$ not significant (pre-operative being $16.3 \pm 3.3)$. IOP on further follow ups at Day 7

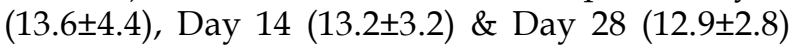
was significantly lower with $(\mathrm{P}<0.001)$. This depicted that IOP was decreasing after surgery.

In high myopia, we found a higher IOP at day one and lower IOP at 7 and 14 days compared with IOP before surgery with no statistic difference $(P=0.49, P=0.57$, and $P=0.13$, ) With a considerably reduced 28-day IOP than before surgery $(\mathrm{P}<.001)$, respectively. And no significant difference was found between day 1 and 7, 7 and 14 days, and 14 and 28 days $(P=0.423, P=0.739$, and $P=0.359$, respectively).

Myopic and emmetropic patients showed almost same trend as mentioned above. In high myopes, IOP rose at day one \& decreased in follow-up sessions but didn't reach the statistical significant 
IOP \& refractive error indices post Cataract Surgery

Table-I: Correlation between refractive errors and change in IOP.

\begin{tabular}{lcccc}
\hline Refractive error & IOP at day 1 & IOP at day 7 & IOP at day 14 & IOP at day 28 \\
\hline Emmetropes & $16.3 \pm 3.3$ & $13.6 \pm 4.4$ & $13.2 \pm 3.2$ & $12.9 \pm 2.8$ \\
Mild Myopes & $15.8 \pm 2.7$ & $14.4 \pm 2.3$ & $13.9 \pm 1.9$ & $13.2 \pm 1.5$ \\
High Myopes & $15.2 \pm 2.9$ & $16.4 \pm 2.6$ & $14.6 \pm 2.8$ & $13.1 \pm 1.8$ \\
\hline
\end{tabular}

values. Among the various groups of refractive errors, high myopes had raised IOP in comparison to others. The changes in IOP at day 1,7,14 and 28 are shown in Table-I.

\section{DISCUSSION}

In this study, we have traversed the relationship between various refractive conditions to postsurgical IOP. We found that cataract surgery with phacoemulsification and intra ocular lens implantation resulted in $87 \%$ success for achieving the goal of decreased IOP. ${ }^{10}$

In a previous study ${ }^{11}$ similar to this, it was depicted that IOP is reduced in post cataract surgeries in glaucoma as well as non-glaucoma patients, due to deepening in the anterior chamber by implantation of IOL, however, which not was congruous to our study.An elevation of $3-4 \mathrm{~mm}$ $\mathrm{Hg}$ was noticed in the immediate post-operative period, it was also well demarcated in previous studies that there is an elevation initiated at three hours and peaked after 24 hours. ${ }^{12,13}$ In a relevant study by Bonnell et al., a significant elevation (10$20 \mathrm{~mm} \mathrm{Hg}$ ) in IOP 24 hours post-operative was narrated. ${ }^{14}$ In comparison, our study exhibited a slight increase in the IOP $(3-4 \mathrm{~mm} \mathrm{Hg})$ at day one that might be due to post-operative inflammation reaction and blockage of trabecular meshwork ${ }^{15}$ by viscoelastic and left out cortical material. ${ }^{16,17}$ Furthermore, emmetropes and mild myopes showed almost same results. There was a linear decrease of IOP in first 14 days as analyzed by change in IOP in these groups and remained persistent for upcoming days. A total of $2-3 \mathrm{~mm}$ $\mathrm{Hg}$ was however reduced which was found to be insignificant.

In candidates with high myopia, there wasn't any manifest change in IOP up till third follow up, after which an instable decline was seen. The reason behind indifferent values of IOP in high myopes might be due to anatomical change that includes raise in visual axis ${ }^{18}$, thinning of choroid and retina, enlarged optic disc and peri-papillary atrophy. ${ }^{19}$ These manifestations exist even after phacoemulsification, thus giving contradictory effects to post-operative lowering of IOP. This phenomenon is the evidence of greater tendency of post-operative IOP fluctuation in high myopia patients that was in harmony to previous studies ${ }^{20}$ and this may be the risk factor for worsening of glaucoma. ${ }^{21}$

Limitations of the study: This study shows the relationship between phacoemulsification and post-operative change in IOP. Our study has few limitations. Firstly, protocol of evaluation of chamber angle configuration was missing. Secondly, due to non-cooperation of few patients, follow up data was lost. Thirdly, being retrospective analysis, we lacked proper data. In synopsis, cataract surgery has an impact on IOP irrespective of the refractive conditions.

\section{CONCLUSION}

Our analysis inferred that the emmetropic, mild to moderate myopic and high myopic patients that underwent phacoemulsification showed depression in IOP at the end of trial follow up, depicting that cataract surgery decreases the IOP regardless of refractive conditions with postoperative time elongation. Hence, cataract surgery does leads to decrease in IOP irrespective of refractive error, though changes are insignificant.

\section{Conflict of Interest: None.}

Grant Support E Financial Disclosures: None.

\section{REFERENCES}

1. Kessel L, Anderson J, Erngaard D, Flesner P, Tendal $\mathrm{B}$, Hjartdal J. Indication for cataract surgery. Do we have evidence of who will benefit from surgery? A systemic review and meta-analysis. Acta Opthalmol. 2016;90(1):10-20.

2. Pascolini D, Mariotti SP. Global estimates of visual impairment: 2010. Br J Ophthalmol. 2012;96:614-618.

3. Javitt JC, Brenner MH, Curbow B. Outcomes of cataract surgery: Improvement in visual acuity and subjective visual function after surgery in the first, second, and both eyes. Arch Ophthalmol. 1993;111:686-691. 
4. Melancia D, Abega Pinto L, Maxques-Never C. Cataract Surgery and Intraocular Pressure. Opthalmic Res. 2015;53:141-148.

5. Shrivastava A, Singh K. The effect of cataract extraction on intraocular pressure. Curr Opin Ophthalmol 2010;21:118-122.

6. Azuara-Blanco A, Burr J, Ramsay C. Effectiveness of early lens extraction for the treatment of primary angle-closure glaucoma (EAGLE): A randomised controlled trial. Lancet. 2016;388:1389-1397.

7. Guan H, Mick A, Porco T, Dolan BJ. Preoperative factors associated with IOP reduction after Cataract Surgery. Optom Vis Sci. 2013;90(2):179-184.

8. Yang HS, Lee J, Choi S. Ocular biometric parameters associated with intraocular pressure reduction after cataract surgery in normal eyes. Am J Ophthalmol 2013;156:89-94.

9. Pruett RC. Progressive myopia and intraocular pressure: What is the linkage? A literature review. Acta Opthalmol Suppl. 1988;185:117-127.

10. Alasbali T, Lofty N M, Al-Gehaban SS, Alkuraya HM, Alsharif A, Khandekar R. Cataract Surgery Audit at a Private Hospital in Saudi Arabia. Middle East Afr J Opthamol. 2015;22(4):502-507.

11. Liu YC, Wilkins M, Kim T. Cataracts. Lancet. 2017;390:600-612.

12. Bomer TG, Lagreze WD, Funk J. Increased intraocular pressure after cataract extraction-effect of surgical technique, surgical procedure and preventive drug administration. A prospective, randomized double-blind study. Klin Monbl Augenheilkd. 1995;206:13-19.

13. Fang EN, Kass MA. Increased intraocular pressure after cataract surgery. Semin Ophthalmol. 1994;9:235-242.

14. Bonnell LN, Soohoo JR, Seibold LK. One-day postoperative intraocular pressure spikes after phacoemulsification cataract surgery in patients taking tamsulosin. J Cataract Refract Surg. 2016;42:1753-1758.
15. Wang N, Chintala SK, Fini ME, Schuman JS. Ultrasound activates the TM ELAM-1/IL-1/NF-KB response: A potential mechanism for intraocular pressure reduction after phacoemulsification. Invest Ophthalmol Vis Sci. 2003;44:1977-1981

16. Tennen DG, Masket S. Short-and long-term effect of clear corneal incisions on intraocular pressure. J Cataract Refract Surg. 1996;22:568-570.

17. Morgan RK, Skuta GL. Viscoelastic-related glaucomas. Semin Ophthalmol 1994;9:229-234.

18. Zhang J, Zou X, Zeng D, Chen W, Sun A, Luo L. Effect of lens constant optimization on the accuracy of intraocular lens power calculation formulas for highly myopic eyes. Int J Opthalmol. 2019;12(6):943-948.

19. Cho BJ, Shin JY, Yu HG. Complications of pathologic myopia. Eye Contact Lens. 2016;42:9-15.

20. Yang Y, Li Z, Wang N. Intraocular pressure fluctuation in patients with primary open-angle glaucoma combined with high myopia. J Glaucoma. 2014;23:19-22.

21. Leidl MC, Choi CJ, Syed ZA. Intraocular pressure fluctuation and glaucoma progression: what do we know? Br J Ophthalmol. 2014;98:1315-1319.

\section{Author`s Contribution:}

MAB formulated, designed and did editing of manuscript.

AAB, AM and NT did data collection, statistical analysis and manuscript writing.

AAB did review, final approval of manuscript and is responsible for integrity of research. 